\title{
Asymmetric Bellow Flexible Pneumatic Actuator for Miniature Robotic Soft Gripper
}

\author{
Ganesha Udupa, ${ }^{1}$ Pramod Sreedharan, ${ }^{1}$ P. Sai Dinesh, ${ }^{2}$ and Doik Kim ${ }^{3}$ \\ ${ }^{1}$ Department of Mechanical Engineering, Amrita Vishwa Vidyapeetham (Amrita University), Kollam, Kerala 690525, India \\ ${ }^{2}$ Department of Electronics and Communication, Amrita Vishwa Vidyapeetham (Amrita University), Kollam, Kerala 690525, India \\ ${ }^{3}$ Interaction \& Robotics Research Center, Korea Institute of Science and Technology, Seoul 136-791, Republic of Korea
}

Correspondence should be addressed to Ganesha Udupa; ammasganesh@gmail.com

Received 23 May 2014; Revised 24 September 2014; Accepted 23 October 2014; Published 3 December 2014

Academic Editor: Anthony A. Maciejewski

Copyright (c) 2014 Ganesha Udupa et al. This is an open access article distributed under the Creative Commons Attribution License, which permits unrestricted use, distribution, and reproduction in any medium, provided the original work is properly cited.

\begin{abstract}
The necessity of the soft gripping devices is increasing day-by-day in medical robotics especially when safe, gentle motions and soft touch are necessary. In this paper, a novel asymmetric bellow flexible pneumatic actuator (AFPA) has been designed and fabricated to construct a miniaturised soft gripper that could be used to grip small objects. The model of AFPA is designed using solid works and its bending motion is simulated in Abaqus software for optimisation and compared with experimental results. The actuator is fabricated using compression molding process that includes micromachining of the molds. Experiments conducted show the bending characteristics of the actuator at different pressures. The actuator shows excellent bending performance and the eccentricity in its design supports increased bending or curling motion up to a certain extent compared to normal bellows without eccentricity. The effects of profile shape and eccentricity on the actuator performance are analysed and the results are presented.
\end{abstract}

\section{Introduction}

Different types of soft actuators are developed that could generate the bending motion by themselves or by the mechanism that bends, due to their actuation $[1,2]$. But most of these actuators are not single chamber or the actuator as a single cannot bend, but a combination of them with appropriate mechanism does the necessary bending motion [3-5]. Flexible pneumatic actuator (FPA) was designed by Joseph L. McKibben in the 1950s known as pneumatic muscle actuator. Toshiba Corp. (Japan) developed a three-degree-of-freedom actuator known as flexible microactuator (FMA) [6]. Even though the FMAs with two or more chambers with fiber reinforcement provide multiple DOF, they require multiple pressure supplies, valves, and sensors as well as complicated manufacturing. Asymmetric flexible pneumatic actuators (AFPAs) have been developed for the first time during the 1990s using asymmetric polymer/rubber tube and rubber bellow actuators with proper reinforcement to overcome the disadvantages of FMA and FPAs and proposed as an innovative method of fabricating a dexterous human hand $[7,8]$. It has also been applied to fabricate a four fingered robot gripper [7-9] and microwalking robot [10]. The design and analysis for application to robotic hand using asymmetric nitrile rubber actuators have been studied [11-13]. Later symmetric thickness bellow actuators for miniature gripper fabricated by moulding technique and rubber bonding process with excimer light $[14,15]$ and asymmetric bellow actuator of about $10 \mathrm{~mm}$ diameter and $120 \mathrm{~mm}$ length that was fabricated by rapid prototyping method to show large bending capabilities [16] are reported. The above group [14, 15] has not worked on the asymmetricity about the longitudinal direction of the actuators. The problem in the symmetric thickness actuator is that it cannot withstand higher pressure ranges. Also the rubber bonding process using ultraviolet light of $172 \mathrm{~nm}$ could be dangerous and expensive. Our technique is further explored and different arrangements of fibers are embedded within the actuator wall [17]. The AFPAs show better deflection up to certain amount of eccentricity provided in the asymmetric actuators. The symmetric bellow actuators [3-6] do not provide larger bending motion and do not withstand high pressures as compared to AFPA. It is mentioned that, in the case 


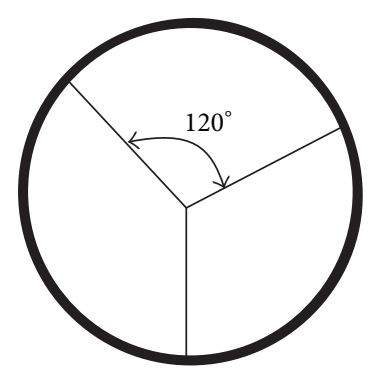

(a)

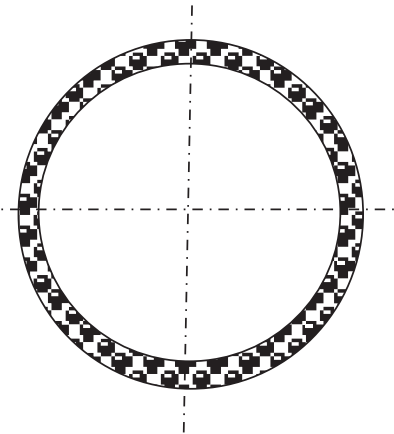

(b)

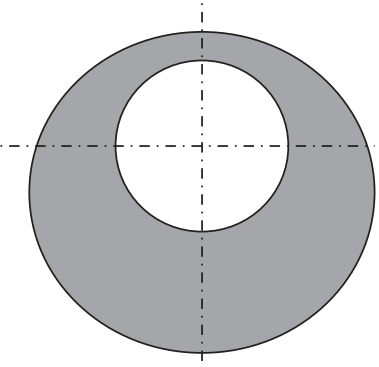

(c)

FIgURE 1: Cross section design of flexible pneumatic actuators (a) FMA, (b) FPA, and (c) AFPA.

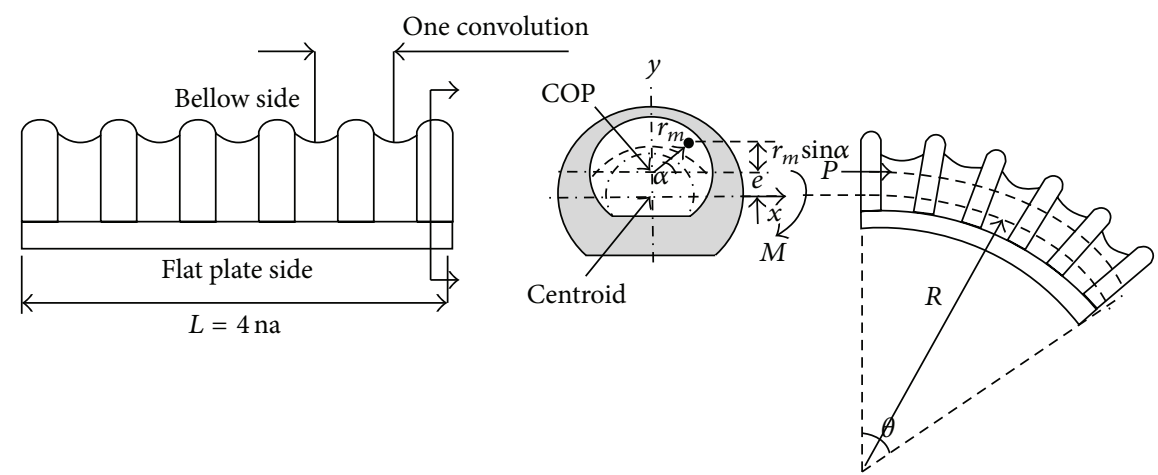

FIGURE 2: Bending of asymmetric bellow actuator subjected to internal pressure.

of symmetric actuators, there is an unstable phenomenon that occurs while gripping when the pressure of the working fluid reaches some limit [3-6]. In this paper, an attempt has been made in the design and fabrication of miniature soft gripper based on the principle of AFPA. The effect of profile shape and determining the optimum eccentricity to get the maximum deflection is analysed using Abaqus 6.13 software. The AFPAs are made almost semicircular in shape with about $4 \mathrm{~mm}$ radius and $30 \mathrm{~mm}$ length. The design idea includes the bellow structure on one side of the actuator and flat side on the other side. The design shape also helps in holding the small objects on flat side of the actuator. For the fabrication, the actuator design is symmetrically divided into two equal halves. Then the manufacturing of each part is done by compression molding process after making the required mold by micromachining process. Then the two-half parts are bonded using single component room temperature vulcanising (RTV) silicone rubber. Experiments are conducted with the developed actuator, by making a miniaturised soft gripper for holding small parts. AFPAs are developed as an alternative to other actuation principles of today, such as electromotors, shape memory alloys, McKibben muscles, and flexible fluidic or pneumatic actuators. These actuators have various advantages considering several criteria, including stress improvements, packaging, good power to weight ratio, and high dynamics. The cross section of AFPA is asymmetric as compared to symmetric section of FMA or FPA as shown in Figure 1.

\section{Theory}

The principle of working of asymmetric bellow actuator is exactly opposite to that of the principle on which the Bourdon tube is working. The Bourdon tube used is initially in curved form with flat or elliptic cross section which under the application of internal pressure will try to straighten up because of the action of the flat or elliptic section becoming circular under pressure. Contrary to this a straight asymmetric (eccentric) bellow tube with circular or semicircular cross section under the application of pressure will become curved and elliptic in cross section. Asymmetric bellow actuator will behave similar to the asymmetric tube actuators [8-10] but will have higher flexibility and greater rate of expansion and curving under internal pressure. Bellows can be made asymmetric in cross section with either circular bellow or semicircular bellow. Figure 2 shows bending of asymmetric flexible semicircular bellow actuator subjected to internal pressure.

When fluid pressure $P$ is applied with the free end closed, it bends due to combined effect of an end moment which develops at the free end due to eccentricity and due to differential expansion of the top and bottom fibers. The force generated due to pressure $P$ is given by $F=P \cdot A_{i}$, where $A_{i}$ 


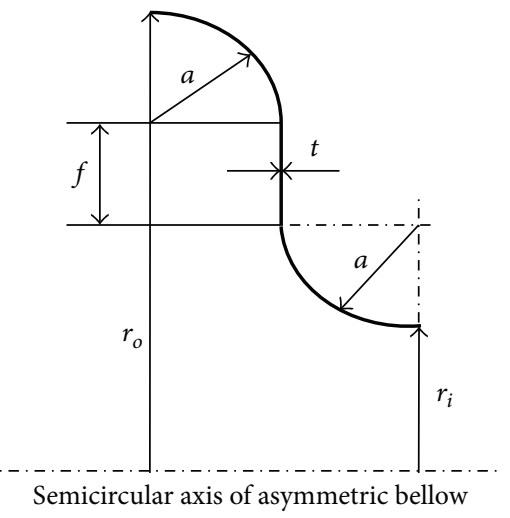

(a)

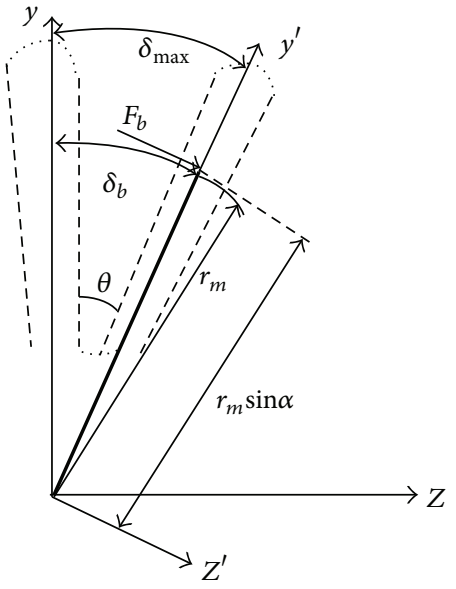

(b)

FIgURE 3: Geometry description of (a) one bellow convolution (b) during bending.

is the internal cavity's area. Since the cut-section is not axissymmetrical, its centroid is slightly shifted from the center of pressure (COP) by a small distance " $e$." Radius of curvature at any pressure is $R=E I / M$ and the bending curvature is defined as $C=1 / R$.

$M$ is the moment acting at the free end, $E$ is the young's modulus, and $I$ is the area moment of inertia.

The moment due to eccentricity of the actuator is $M_{e}=$ $F \cdot e$, where $e$ is the eccentricity.

The deflection of the actuator is due to differential expansion and the moment created due to the eccentricity of the geometry [7-9]. From the beam theory, the vertical deflection $\delta_{0}$ at the tip of the bellow and the angular deflection $\theta_{1}$ are given in terms of $M$ as

$$
\begin{gathered}
\delta_{0}=\frac{M L^{2}}{3 E I_{y}}, \\
\theta_{1}=\frac{M L}{E I_{y}},
\end{gathered}
$$

where $I_{y}$ is the area moment of inertia of the cross section of asymmetric bellow and $L$ is the length of the bellow.

In the case of differential expansion for the bellow, it is considered to have separate stiffness constant " $k$ " for the top and plate side of the bellow. The force applied to the ABA is approximated to axial force and separated into $F_{b}$ and $F_{p}$, respectively, for both sides of the bellow.

The total force is given by

$$
F=F_{b}+F_{p}=k_{b} \delta_{b}+k_{p} \delta_{p},
$$

where $\delta_{b}$ and $\delta_{p}$ are the deflections at the top bellow side and bottom flat part side, respectively. The $k_{b}$ and $k_{p}$ are the axial stiffness of the top bellow side and bottom flat part side, respectively.

The axial stiffness, $k_{b}=B I_{y}$, is formulated using finite element and linear regression analysis [18] with modifications for semicircular asymmetric bellow. The simplified geometry description of one bellow convolution is shown in Figure 3.
$B$ is constant which depends on the geometry and the material of the bellow and is given by

$$
\begin{gathered}
B=\frac{24 E\left(4.602+6 \times 10^{7} a^{3}-86.2 r_{0}\right)}{4 n\left\{6 \pi a^{3}+24 f a^{2}+f^{3}+3 f^{2} a \pi\left(1+t^{2} / 12 a^{2}\right)\right\}}, \\
I_{y}=\frac{\pi\left(r_{0}+r_{i}\right) t^{3}}{12}=\frac{\pi r_{m} t^{3}}{6},
\end{gathered}
$$

where $a$ is radius of corrugation, $f$ is flank distance, $t$ is bellow thickness, $r_{0}$ and $r_{i}$ are outer and inner radius of the bellow, $r_{m}$ is the average radius of the bellow, $E$ is modulus of elasticity, and $n$ is number of convolutions of ABA.

Thus deflection at bellow (top) side of the ABA is

$$
\delta_{b}=\frac{F_{b}}{k_{b}} .
$$

The deflection in the bottom plate part will be

$$
\delta_{p}=\frac{F_{p}}{k_{p}} .
$$

Thus the angular deflection is given by

$$
\theta_{2}=\frac{\delta_{b}-\delta_{p}}{r_{m}}=\frac{\delta_{b p}}{r_{m}} .
$$

The total angular deflection of the asymmetric bellow actuator due to bending is obtained by adding (2) and (8) and is given by

$$
\theta=\theta_{1}+\theta_{2}=\frac{M L}{E I_{y}}+\frac{\delta_{b p}}{r_{m}} .
$$

The moment due to differential expansion of the bellow and plate part of the bellow is assumed to be acting at the semicircular axis of the asymmetric bellow section and the maximum deflection; $\delta_{\max }$ is at the top of the bellow. 
To determine the moment to bend the bellow due to differential expansion, the deflection force which varies around the circumference will be multiplied by the corresponding levers and integrated around the circumference.

From (6), $F_{b}=k_{b} \delta_{b}=B I_{y} \delta_{b}$.

Assuming an element force, $d F$ at any location at the average radius of the bellow is

$$
d F=\delta_{b} B I_{y}\left(\frac{d \alpha}{\pi}\right) .
$$

For maximum deflection and substituting (5) into (10),

$$
d F=\delta_{\max } B r_{m} \frac{t^{3}}{6} \sin \alpha d \alpha
$$

The element moment is

$$
d M=d F r_{m} \sin \alpha=\frac{\delta_{\max } B t^{3} r_{m}^{2}}{6} \sin ^{2} \alpha d \alpha .
$$

The moment due to expansion of the bellow actuator is given by

$$
M_{\exp }=\frac{\delta_{\max } B t^{3} r_{m}^{2}}{6} \int_{0}^{\pi} \sin ^{2} \alpha d \alpha=\frac{\delta_{\max } B \pi t^{3} r_{m}^{2}}{12}=\frac{F_{\max } r_{m}}{2},
$$

where $F_{\max }$ is the maximum force at the top of the bellow where it is subjected to maximum deflection. by

The total moment due to internal pressure $P$ is obtained

$$
M=F \cdot e+\frac{F_{\max } r_{m}}{2} .
$$

\section{Design and Analysis}

The design of the AFPA is almost semicircular in shape such that on one half it has a bellow profile and on the other side it is flat. This actuator is a single chambered tubular structure, whose thickness of one side is more than the thickness of other side. The actuator bends towards the thicker side due to the differential expansion of top and bottom parts of the asymmetric bellow and moment generated due to eccentricity of the bellow. The asymmetric bellow design gives maximum deflection compared to normal symmetric designs up to certain value of eccentricity and withstands high pressures. The shape of the bellow profile also affects the deflection of the actuator. Figure 4 shows various bellow shapes. The percentages of expansion when the internal pressure is increased from $P_{0}$ to $P_{1}$ are $26,47,48$, and 70 for triangular, trapezoidal, $U$ shape, and square shapes, respectively. The square shaped bellow profile is more suitable as it gives maximum deflection. To decide on an optimum design for the efficient actuation, the FE analysis is done on Abaqus 6.13 using models with different dimensions. The material used for the actuator is a two-component silicone rubber which is RTV (room temperature vulcanising) type (KE-1606, ShinEtsu Silicones Corp.). Table 1 shows the material properties of the silicone rubber used in analysis and manufacturing.

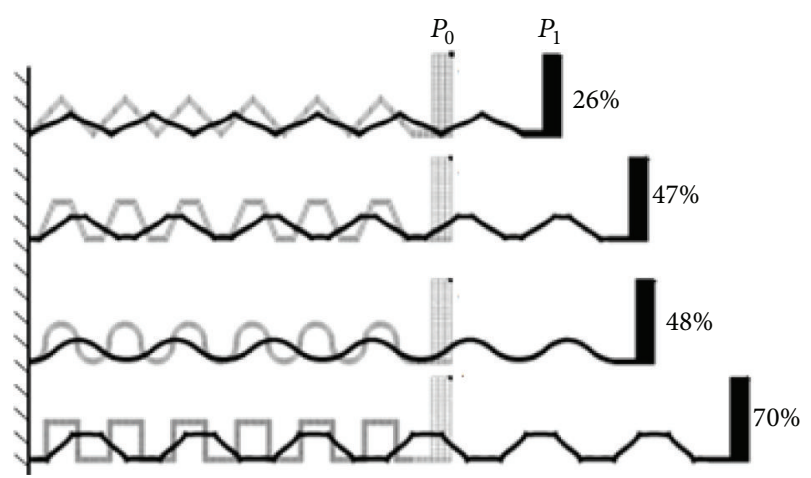

FIGURE 4: Effect of bellow shape on the expansion of actuator.

TABLE 1: Material properties of silicone rubber.

\begin{tabular}{lcc}
\hline & Properties & Values \\
\hline 1 & Appearance & Translucent \\
2 & Density & $1.03 \mathrm{~kg} / \mathrm{m}^{3}$ \\
3 & Shore hardness & 28 \\
4 & Tensile strength & $4.3 \mathrm{MPa}$ \\
5 & Tear strength & $12 \mathrm{kN} / \mathrm{m}$ \\
6 & Elongation & $350 \%$ \\
\hline
\end{tabular}

TABLE 2: Parameters of AFPA.

\begin{tabular}{lcc}
\hline Model type & $A[\mathrm{~mm}]$ & $B[\mathrm{~mm}]$ \\
\hline Model 1 & 0.7 & 0.7 \\
Model 2 & 0.9 & 0.7 \\
Model 3 & 1 & 0.7 \\
Model 4 & 1.1 & 0.7 \\
Model 5 & 1.5 & 0.7 \\
\hline
\end{tabular}

Figure 5 shows the model of the AFPA with dimensions created in the CAD software. To find the optimum design, different models with varying parameters $A$ and $B$ are created for analysis which are responsible for the eccentric actuation. $A$ and $B$ represent the thickness of flat plate side and the thickness of bellow side, respectively. As shown in Table 2, model 1 to model 5 with different values of parameters are considered for analysis. Length of the actuator in all models is $30 \mathrm{~mm}$ and radius is about $3 \mathrm{~mm}$.

Figure 6 shows the analysis results for the deflection of AFPA. The deflection is analysed using commercial finite element code Abaqus 6.13. Five models shown in Table 2 are used to measure the deflection with respect to the pressure applied. The asymmetric model 3 shows better deflection compared to the other models. In model 1, the thicknesses of both flat plate side and bellow side are the same. Model 5 , where the thickness of the flat plate side is twice the thickness of bellow side, gives less deflection compared to all the other models. Therefore it is observed that the deflection of asymmetric bellow actuator is influenced by the eccentricity provided up to a certain extent. This shows that as the eccentricity increases by increasing the thickness of the flat plate side, the stiffness also increases which reduces 


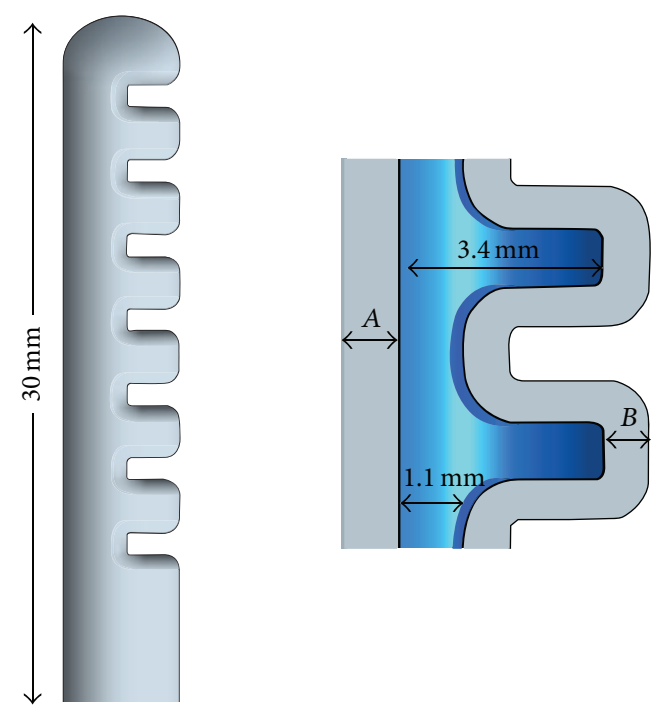

Figure 5: CAD model of the AFPA.

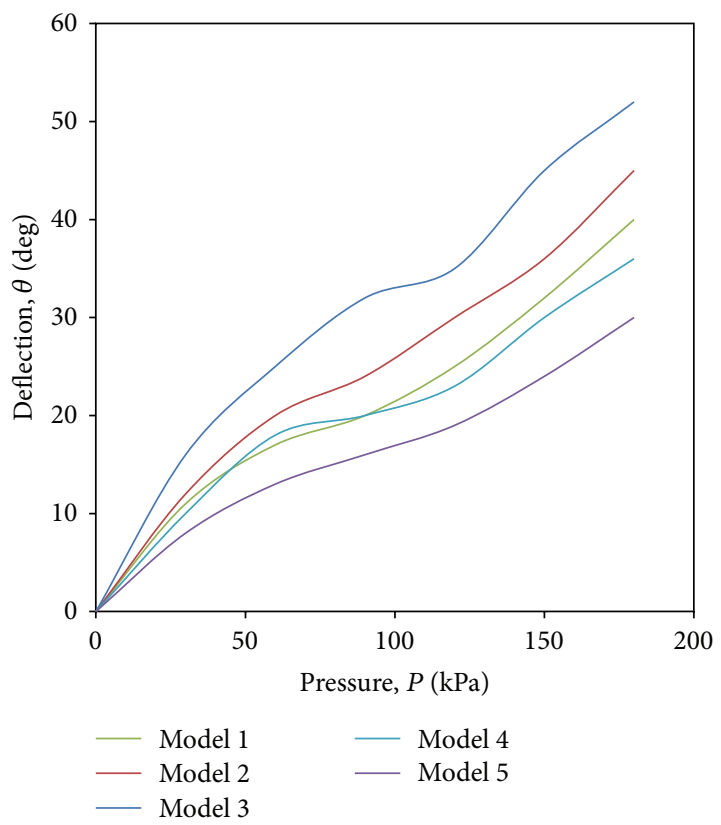

FIGURE 6: Deflection of AFPA for various models.

the deflection of the actuator considerably. In this paper, model 3 is chosen for manufacturing the actuator.

Figure 7 shows the variation of bending angle with respect to flat plate thickness $(A)$. The bending angle increases as the flat plate thickness increases up to $1 \mathrm{~mm}$. After $1 \mathrm{~mm}$ of flat plate thickness of the bellow part, the bending angle starts decreasing. This means that there is an optimum bending angle for certain amount of eccentricity of the actuator. As the flat plate thickness of bellow actuator increases, the stiffness also increases leading to decrease in bending angle.

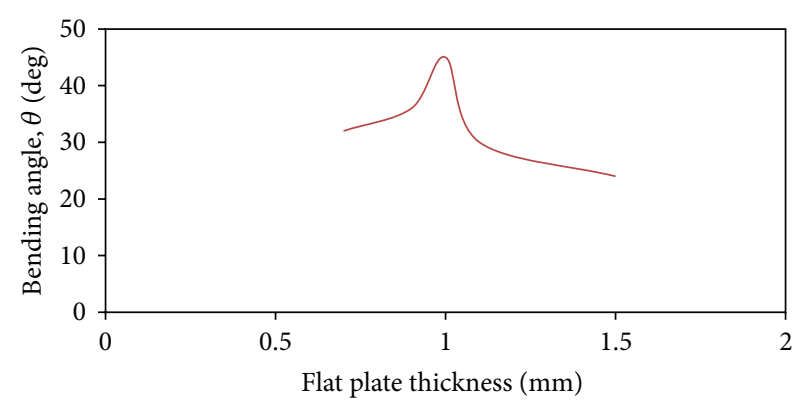

FIGURE 7: Variation of bending angle for different flat plate thickness $(A)$ of actuator.

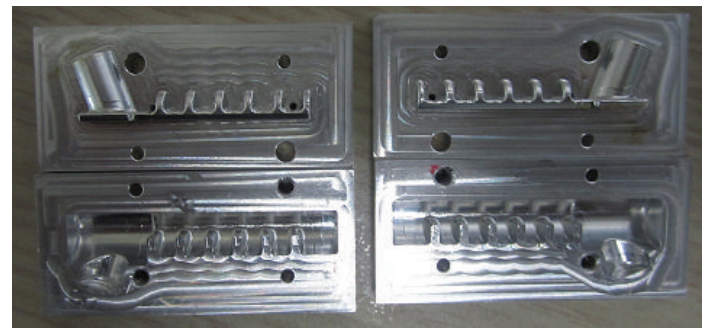

Figure 8: Fabricated convex and concave mold parts of AFPA.

\section{Manufacturing of AFPA}

The AFPA is manufactured using compression molding technique. For the manufacturing purpose, the actuator is divided into two symmetrical equal halves along the axis. Then each half is manufactured separately and is bonded to make the complete AFPA. The manufacturing of each half part involves machining process for molds and preparation of the silicone rubber paste for molding. Initially with appropriate dimensions from the simulations molds are manufactured using machining process, which includes the convex and concave part of the mold. Figure 8 shows the mold manufactured using micromachining process. This mold can be used to manufacture half part of the actuator and similarly other two molds are made for the other half part of AFPA.

Now, for the preparation of the silicone rubber paste, whose properties are as shown in Table 1, KE-1606 RTV silicone rubber is mixed with a $10 \%$ of curing agent (CAT-RG). Then this paste is poured between the convex and concave mold for both half parts of the actuator and then locked for curing. After curing, both the parts are bonded using onecomponent RTV silicone rubber. The advantage in using onecomponent silicone rubber as bonding agent is that after curing its property of flexibility remains as compared to other adhesive materials, which makes the AFPA flexible as a single continuous piece. Figure 9 shows the manufactured half parts of the AFPA after molding process and the complete actuator after bonding the two half parts along with a pressure inlet.

One-component Shin-Etsu KE 45 room temperature vulcanizing (RTV) silicone rubber compound is used for chemically bonding the two silicon bellow parts. Shin-Etsu KE45 RTV adhesive is a paste-like, one-component material that 


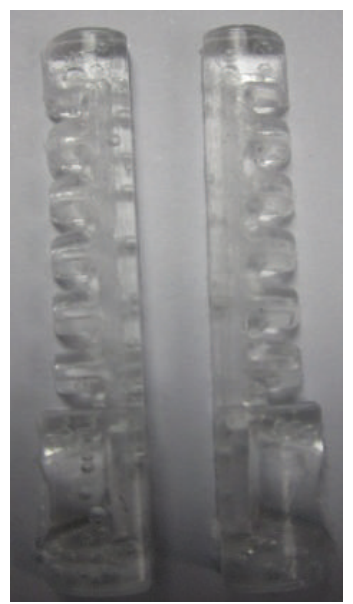

(a)

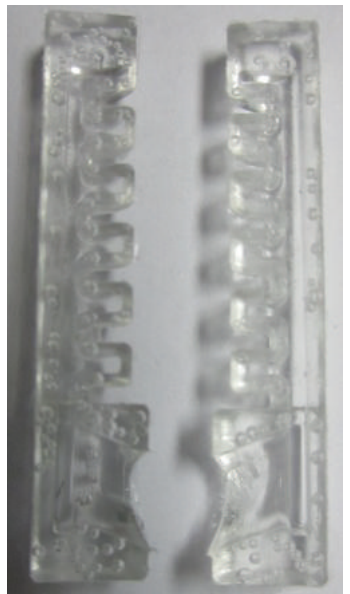

(b)

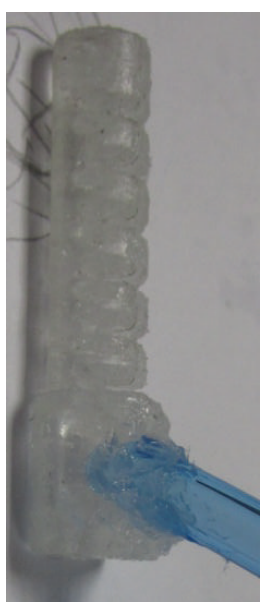

(c)

FIgURe 9: Manufactured parts of AFPA: (a) outside view, (b) inside view, and (c) complete actuator.

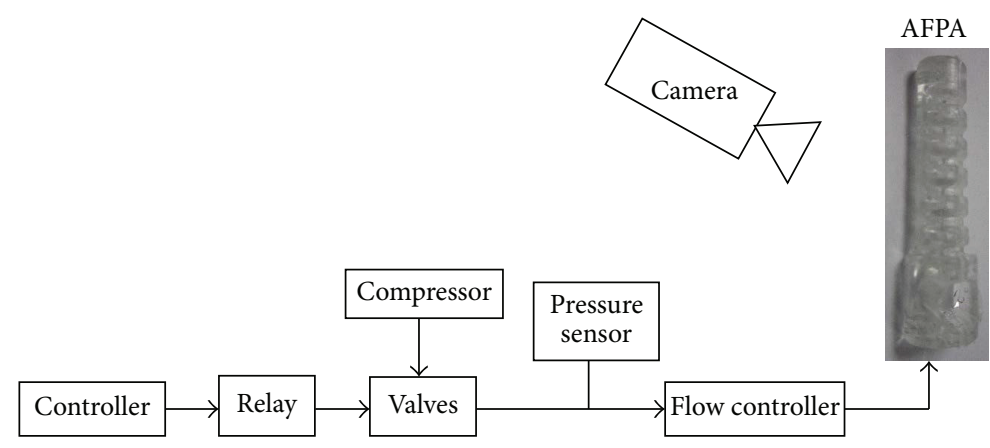

FIGURE 10: Block diagram of experimental setup for the control of AFPA.

cures when exposed to moisture in the air at room temperature. Due to its nonsag, nonflowable features, it may be applied overhead or on side wall joints or surfaces. The bonding process involves first cleaning the rubber bellow parts and degreasing if necessary. Apply the silicone rubber 1component adhesive (KE $45 \mathrm{RTV}$ ) to a thickness of at least $0.5 \mathrm{~mm}$. Press together the bellow parts to be bonded and fix them in position until the adhesive has cured. The curing time is about 10 minutes. Depending on the air humidity, the optimum bond strength at room temperature is achieved within 12 to 15 hours. It is much faster at temperatures between 50 and $100^{\circ} \mathrm{C}$ and high humidity.

\section{Experimental Setup}

Figure 10 shows the block diagram of experimental setup required for the control of the AFPA. Figure 11 shows actual experimental setup showing three AFPAs connected to air pressure supply via valves, pressure sensor, and flow controller. The pressure inside the actuator is controlled by the control of valves using a microcontroller. The motion of the AFPA is captured by a camera.

A 3-port solenoid valve having pressure input, output, and exhaust ports is used in the experimental setup. Input of

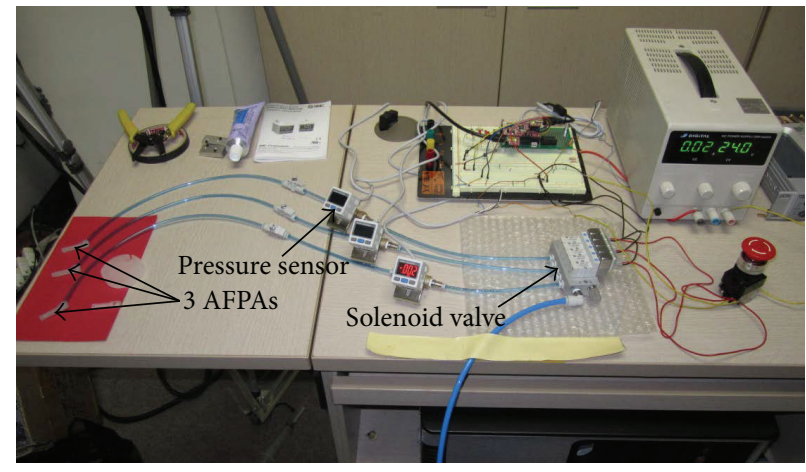

FIGURE 11: Experimental setup showing various devices.

the valve is connected to the compressor output and output of the valve is connected to the actuator input. The principle of 3-port valve is to internally direct the pressure to the AFPA from the compressor and then to the exhaust from the AFPA corresponding to the $\mathrm{ON}$ and OFF state of the valve. The control of the valve is done by the Arm microcontroller via relay circuit as the valve working voltage is $24 \mathrm{~V} \mathrm{DC}$. 


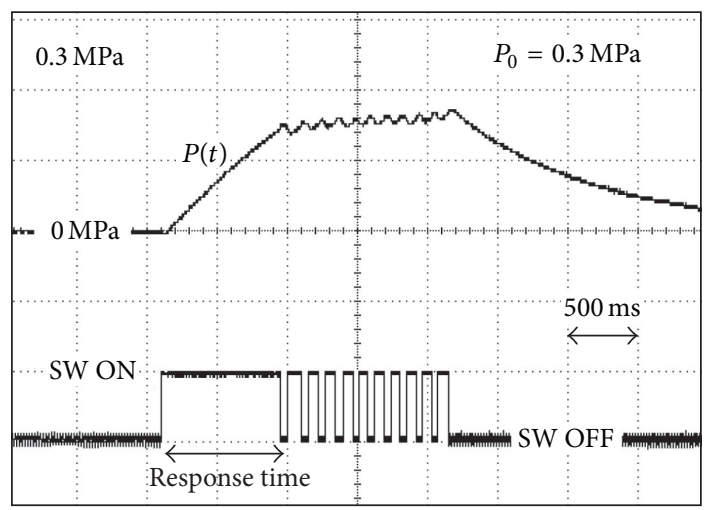

Figure 12: PWM control of the solenoid valve.

Figure 12 shows the graphical relation for input and output of the solenoid valve. When the valve is in ON state, the pressure increases linearly in the AFPA. To maintain the pressure inside the actuator at a constant value (e.g., 0.3 MPa) for a desired position, the valve should be controlled using a PWM pulse with 50\% duty cycle as shown. Stellaris LM3S808 Arm microcontroller is used to generate PWM using 16-bit PWM mode.

The design of the actuator is such that, at a particular pressure, the actuator reaches its maximum deflection, after which any increase in the pressure results in bulging of the actuator. The time taken for the actuator to reach its maximum pressure is called response time. The controller is designed to maintain the maximum safe pressure in the actuator and also to maintain the position of the actuator at the desired point. To achieve this, the ON/OFF control of the solenoid valves should be correspondingly done. The configuration of the valve is such that when the valve switch is ON, the pressure starts increasing and when its switch is OFF, the pressure starts decreasing. As shown in Figure 12, the upper part shows the pressure in the actuator depending on the switching state of the valve as shown in the lower part of the figure. Let the pressure $P_{0}$ shown in Figure 12 be the required pressure for the desired deflection or position of the actuator. To maintain the pressure, the valve should be switched on until the pressure inside the actuator reaches $P_{0}$ after which switching should follow a PWM with 50\% duty cycle and the frequency of $10 \mathrm{~Hz}$ (maximum operating frequency of the solenoid valve). To generate the PWM and to read the pressure value from the pressure sensor, Arm microcontroller is selected. The controller is a 32-bit CPU with operating frequency of $50 \mathrm{MHz}$ which can generate five 16-bit PWM at a time. Figure 13 shows the closed loop feedback control system.

The programming of the controller is carried out using "C" language. Figure 14 shows the flow chart of control program. The $100 \%$ duty cycle implies the ON state of the valve that results in the increase of pressure, $50 \%$ duty cycle implies the switching state of the valve that results in the constant value of pressure, and $0 \%$ duty cycle implies the OFF state of the valve that results in the decrease of pressure.

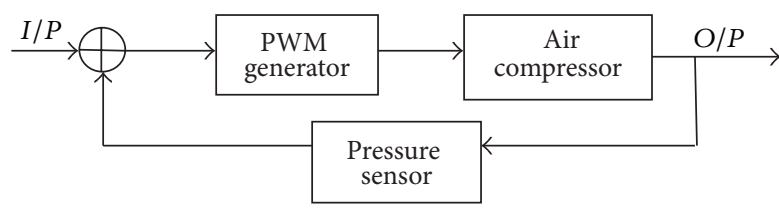

Figure 13: Closed loop feedback control system.

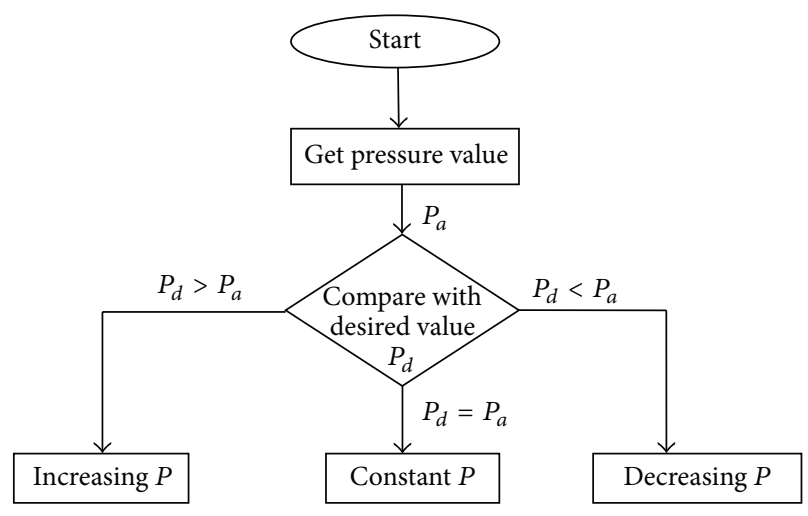

FIGURE 14: Flow chart of control program.

\section{Results and Discussion}

Figure 15 shows the deflection of manufactured AFPA with varying pressures. For every $30 \mathrm{KPa}$ increase in pressure the corresponding deflection of the AFPA is shown. The developed actuator shows a very good position control with the PWM control technique. And also the simple structure of the actuator with single chamber is more easy to be miniaturised compared to the conventional actuators of two or more chambers [3-6].

Figure 16 shows deflection analysis of the AFPA using Abaqus 6.13 software. Hyperelastic tetrahedron elements are used for rubber structures on wall of the actuator. Applied pneumatic pressures are given as incremental pressure load in the software, which always acts in the nominal direction on the rubber walls of the actuator. In the FEM analysis, the Mooney-Rivlin model is used for approximating the characteristics of silicone rubber. The coefficients are identified through the experimental results of plane strain tension tests of the silicone rubber.

The deflection angles are $27^{\circ}, 30^{\circ}, 43^{\circ}$, and $48^{\circ}$ as against the $35^{\circ}, 37^{\circ}, 48^{\circ}$, and $56^{\circ}$ as obtained by the manufactured actuator at internal pressures of $90 \mathrm{kPa}, 120 \mathrm{kPa}, 150 \mathrm{kPa}$, and $180 \mathrm{kPa}$, respectively. There is a slight variation in the analysis results as compared to experimental results. This could be due to error during modelling and analysis in the software. Also since the rubber material is elastic in nature and has highly nonlinear property, it is difficult to analyze large deformations using software. Due to pressure, the rate at which the bending occurs is very high which again is difficult to predict theoretically with high accuracy. 


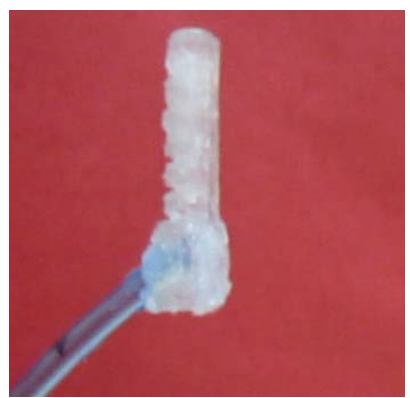

(a) $0 \mathrm{kPa}$

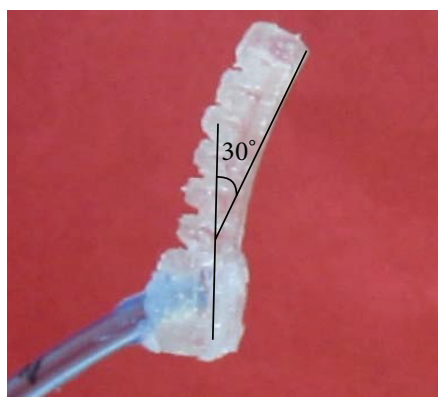

(b) $60 \mathrm{kPa}$

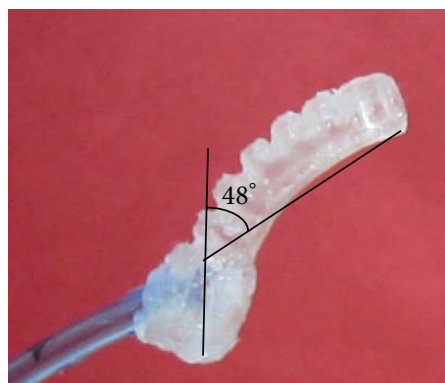

(e) $150 \mathrm{kPa}$

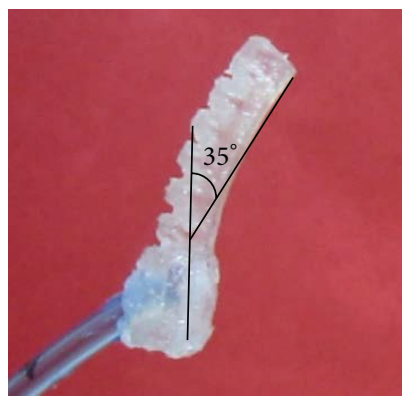

(c) $90 \mathrm{kPa}$

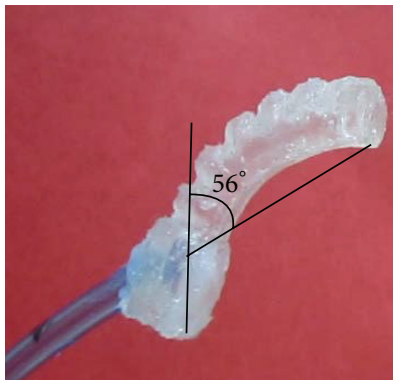

(f) $180 \mathrm{kPa}$

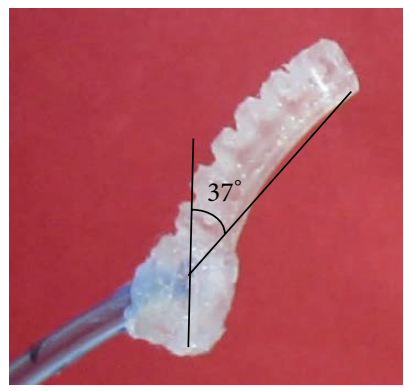

(d) $120 \mathrm{kPa}$

FIgURE 15: Deflection of the AFPA of model 3 at different pressures.

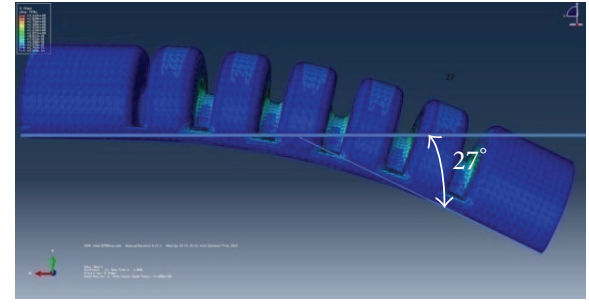

(a)

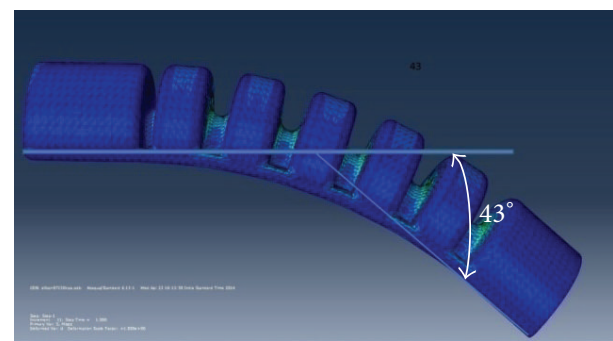

(c)

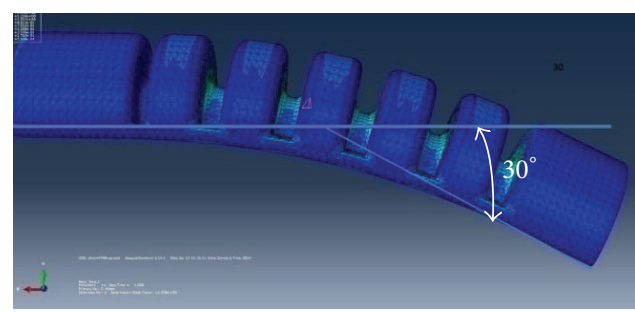

(b)

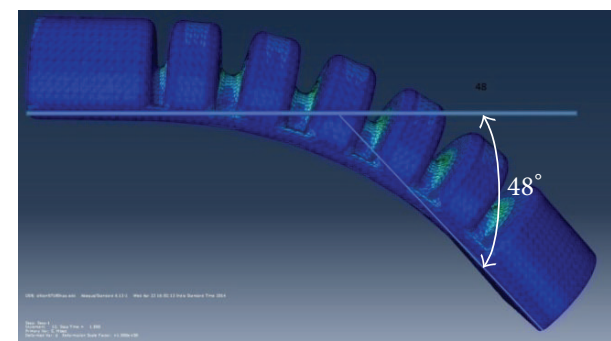

(d)

FIGURE 16: Deflection analysis of model 3 AFPA for various internal pressures (a) $90 \mathrm{kPa}$, (b) $120 \mathrm{kPa}$, (c) $150 \mathrm{kPa}$, and (d) $180 \mathrm{kPa}$.

Figure 17 shows the static bending characteristic of the developed actuator. The analysis and experimental results are close to each other. The simple characteristic equations are obtained by assuming Young's modulus $(E)$ is constant and the calculated characteristics are compared with experimental data taken from a $6 \mathrm{~mm}$ diameter.

Figure 18 shows the path followed by the tip of the actuator at different pressures. The pressure is varied from $0 \mathrm{kPa}$ to $180 \mathrm{kPa}$ insteps of $30 \mathrm{kPa}$. The displacement can be seen in both $x$ and $y$ directions. The displacement in both directions increases up to $150 \mathrm{kPa}$ after which the tip of the actuator starts curling.

The maximum force generated by the actuator is measured by a load cell. To detect the force, one end of the actuator is fixed and the deflecting end is touching the load cell. The load cell is set up to measure the force from the tip of the actuator. Figure 19 shows the experimental and theoretical force curves of the actuator subjected to various 


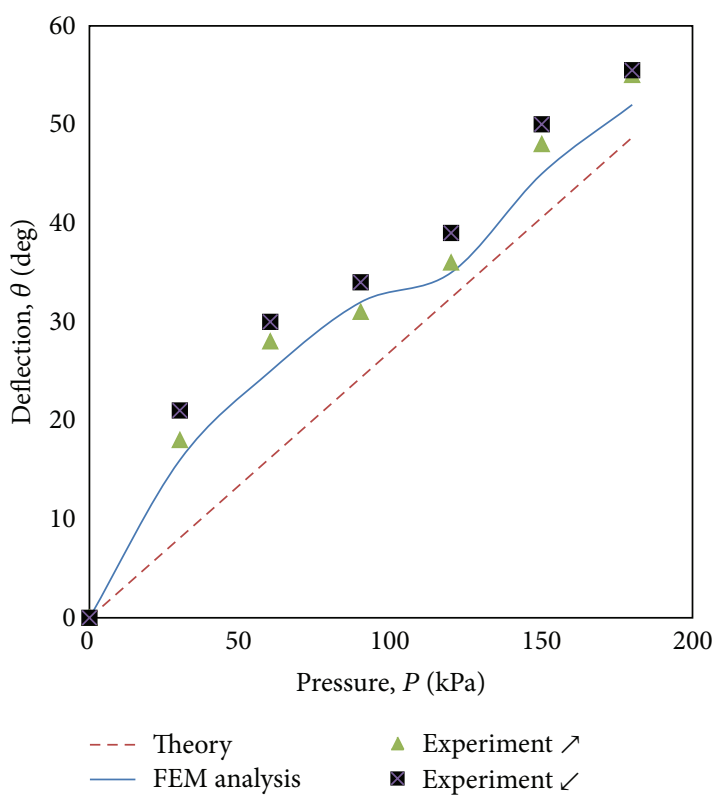

FIGURE 17: The static bending characteristics of AFPA.

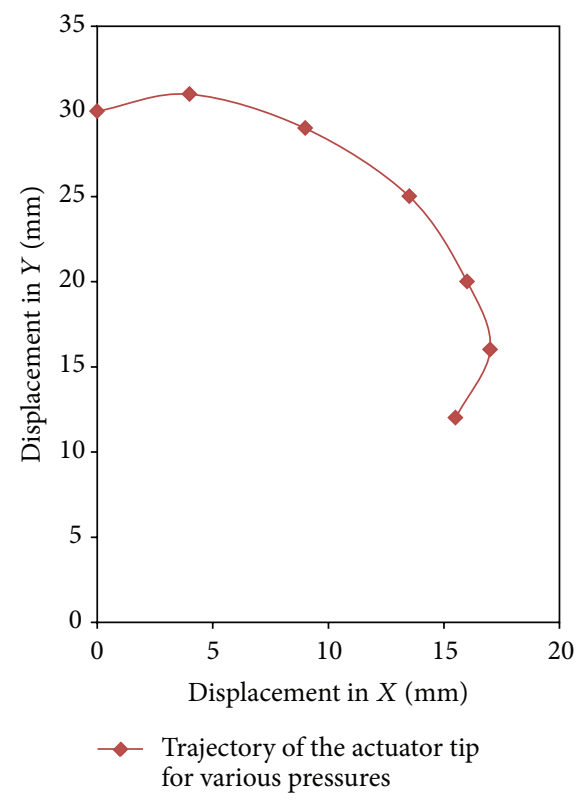

FIGURE 18: Trajectory of the tip motion of the actuator.

internal air pressures. The pressure range is from 0 to $180 \mathrm{kPa}$. The force characteristics are almost linear, and the maximum measured force is $0.17 \mathrm{~N}$ at $180 \mathrm{kPa}$.

\section{Miniature Soft Gripper}

A miniature soft gripper consisting of three AFPAs is constructed to pick and place small parts. These three AFPAs are fixed to a silicone rubber plate at an angle of 120 degrees as shown in Figure 20. Figure 20 shows the various gestures of the miniature soft gripper grasping IC chips. The effect of

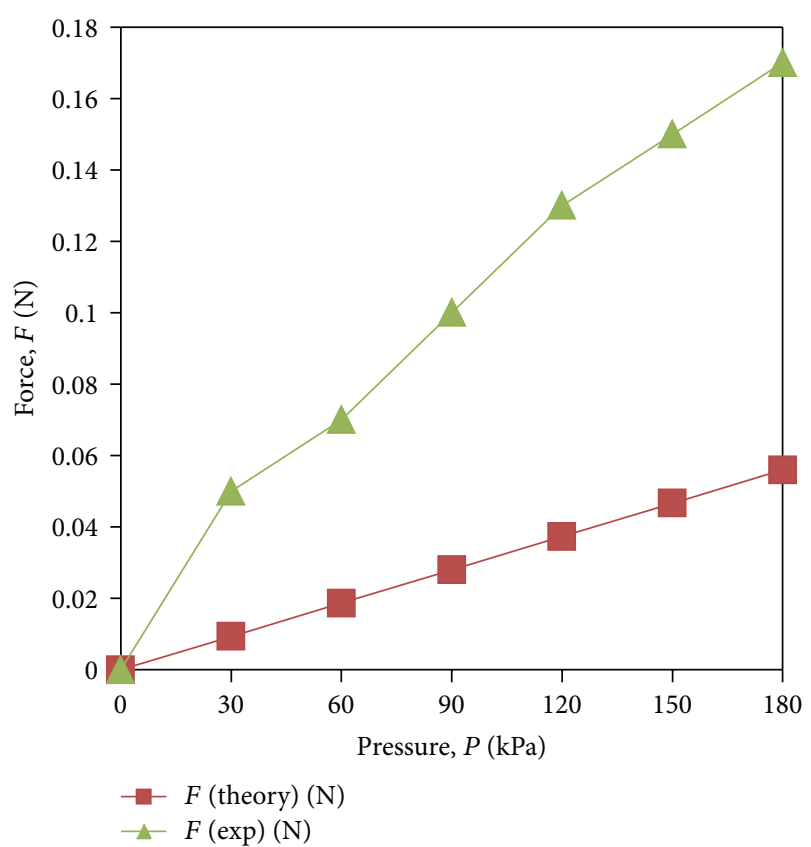

FIGURE 19: Experimental and theoretical results of force measurement.

instability while gripping when the pressure of the working fluid reaches some limits as in the case of symmetric FPAs is avoided.

In the three-chamber design of pneumatic actuators [35], it has been reported that when the pressure of the working fluid reaches some limit, an unstable phenomenon occurs. The object and FMAs turn unstably around the polar axis of the actuator. This is because of a net torque acting about the polar axis due to the differential pressures in the various chambers that causes twisting about the polar axis with possible torsional buckling. This problem is not observed in our design as there is only a single chamber and the pressure in this chamber does not create a net torque about the actuator's polar axis.

\section{Conclusion}

In this paper, a single chamber miniaturised asymmetric flexible pneumatic bellow actuator has been designed and fabricated which gives bending performance better than the symmetric actuators of two or more chambers. Using such three actuators, a miniature soft gripper has also been developed. Analysis in ABAQUS software resulted in optimised design of the actuator. It is found that the effect of shape and eccentricity of the AFPA plays an important role in the bending of the actuator and deflection of asymmetric bellow actuator is influenced by the eccentricity up to a certain extent. The effect of instability while gripping when the pressure of the working fluid reaches some limits as in the case of symmetric FPAs is avoided. The assembled actuator in the form of gripper has shown good results in picking of and placing small parts. It can also be shown that, by supplying vacuum or negative pressure to the actuator, the actuator 


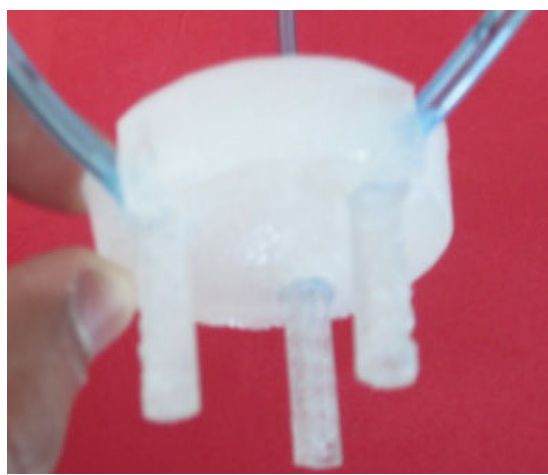

(a)

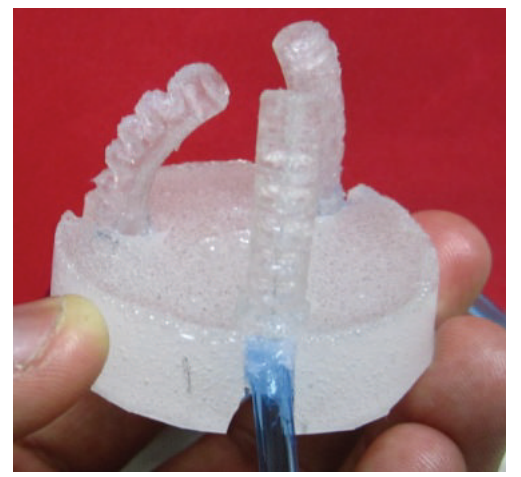

(b)

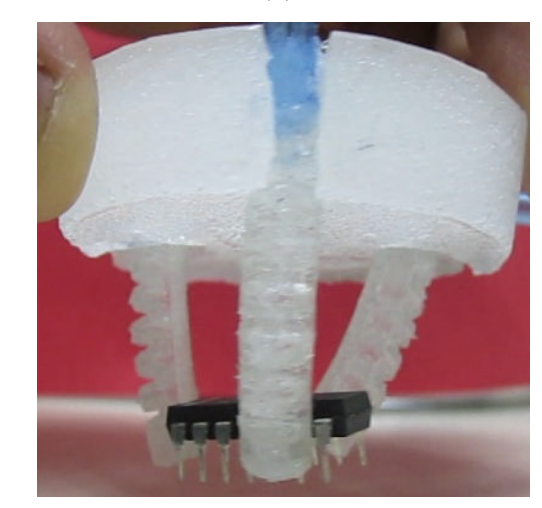

(d)

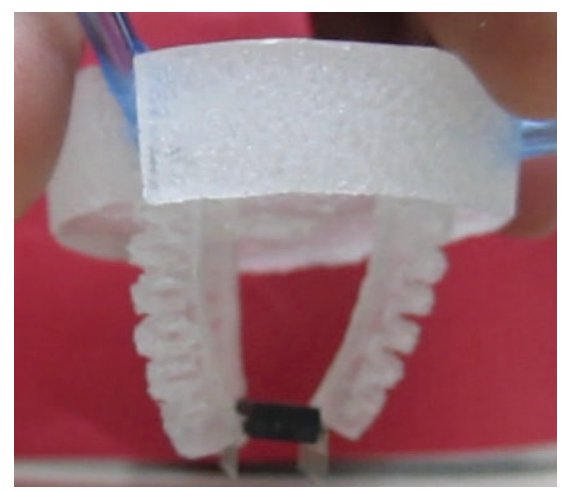

(c)
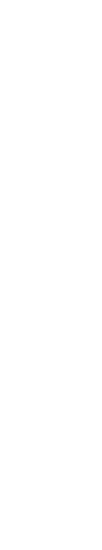

FIGURE 20: Miniature soft grippers: (a) without application of pressure, (b) with application of pressure, (c) grasping IC chip, and (d) grasping up IC chip.

can generate bending motion in counter direction of positive pressure. These types of AFPAs will be useful in miniature robotic mechanisms where space is restricted or gentle handling is required.

\section{Conflict of Interests}

The authors declare that there is no conflict of interests regarding the publication of this paper.

\section{Acknowledgment}

The authors would like to thank KIST (Korea Institute of Science and Technology), Korea, for providing internship to Amrita University students.

\section{References}

[1] L. Zhang, Z. Wang, Q. Yang, G. Bao, and S. Qian, "Development and simulation of ZJUT hand based on flexible pneumatic actuator FPA," in Proceedings of the IEEE International Conference on Mechatronics and Automation (ICMA '09), pp. 1634-1639, IEEE, Changchun, China, August 2009.

[2] I. Gaiser, S. Schulz, H. Breitwieser, and G. Bretthauer, "Enhanced flexible fluidic actuators for biologically inspired lightweight robots with inherent compliance," in Proceedings of the IEEE International Conference on Robotics and Biomimetics (ROBIO '10), pp. 1423-1428, Tianjin, China, December 2010.

[3] K. Suzumori, S. Iikura, and H. Tanaka, "Applying a flexible microactuator to robotic mechanisms," IEEE Control Systems Magazine, vol. 12, no. 1, pp. 21-27, 1992.

[4] K. Suzumori, S. Endo, T. Kanda, N. Kato, and H. Suzuki, "A bending pneumatic rubber actuator realizing soft-bodied manta swimming robot," in Proceedings of the IEEE International Conference on Robotics and Automation (ICRA '07), pp. 4975-4980, Roma, Italy, April 2007.

[5] K. Suzumori, S. Iikura, and H. Tanaka, "Development of flexible microactuator and its applications to robotic mechanisms," in Proceedings of the IEEE International Conference on Robotics and Automation, pp. 1622-1627, Sacramento, Calif, USA, April 1991.

[6] K. Suzumori, S. Iikura, and H. Tanaka, "Flexible microactuator for miniature robots," in Proceedings of the IEEE Micro Electro Mechanical Systems Conference, pp. 204-209, Nora, Japan, 1991.

[7] G. Udupa, Study and development of an unconventional device for industrial applications including robots and instrumentation [M.S. thesis], University B.D.T College of Engineering, Davangere, India, 1992.

[8] G. Udupa and R. Krishna Murthy, "A new flexing technique for soft Gripper design," in Proceedings of the 16th All India Manufacturing Technology Design and Research Conference, pp. 353-358, Bangalore, India, December 1994.

[9] G. Udupa, P. Sreedharan, and K. Aditya, "Robotic gripper driven by flexible microactuator based on an innovative technique," in Proceedings of the 6th IEEE Workshop on Advanced Robotics 
and Its Social Impacts, pp. 1-6, Korean Institute of Science and Technology, Seoul, Korea, October 2010.

[10] S. Dinesh, R. Raveendran, K. Aditya, P. Sreedharan, and G. Udupa, "Innovative micro walking robot using flexible microactuator," in Proceedings of the 28th International Symposium on Automation and Robotics in Construction, Seoul, Republic of Korea, June 2011.

[11] C. P. S. Menon, P. Sredharan, and G. Udupa, "Design and analysis of multi-fingered dexterous hand based on an innovative asymmetric flexible pneumatic actuator," in Proceedings of the 2nd International Conference on Simulation, Modeling, and Analysis, pp. 246-253, Vishwa Vidyapeetham, Coimbatore, India, 2011.

[12] K. B. S. Pavan Kumar, S. K. Srinath, C. P. Sankar Menon, S. Pramod, and G. Udupa, "A novel technique for the development of an artificial human hand for prosthetic application," in Proceedings of the National Conference on Application of Data Mining in Management of Metabolic and Degenerative Disorders, India, pp. 1-7, April 2012.

[13] G. Udupa, "Artificial robotic hand and process of manufacturing thereof," Patent 3631/CHE/2011, 2011.

[14] S. Wakimoto, K. Ogura, K. Suzumori, and Y. Nishioka, "Miniature soft hand with curling rubber pneumatic actuators," in Proceedings of the IEEE International Conference on Robotics and Automation (ICRA '09), pp. 556-561, Kobe, Japan, May 2009.

[15] S. Wakimoto, K. Suzumori, and K. Ogura, "Miniature pneumatic curling rubber actuator generating bidirectional motion with one air-supply tube," Advanced Robotics, vol. 25, no. 9-10, pp. 1311-1330, 2011.

[16] Y. Shapiro, A. Wolf, and K. Gabor, "Bi-bellows: pneumatic bending actuator," Sensors and Actuators, A: Physical, vol. 167, no. 2, pp. 484-494, 2011.

[17] S. Hirai, T. Masui, and S. Kawamura, "Prototyping pneumatic group actuators composed of multiple single-motion elastic tubes," in Proceedings of the IEEE International Conference on Robotics and Automation (ICRA '01), vol. 4, pp. 3807-3812, Seoul, Republic of Korea, May 2001.

[18] M. Hermann and A. Jonsson, Static characteristics of flexible bellows [M.S. thesis], University of Karlskrona, Karlskrona, Sweden, 1997. 

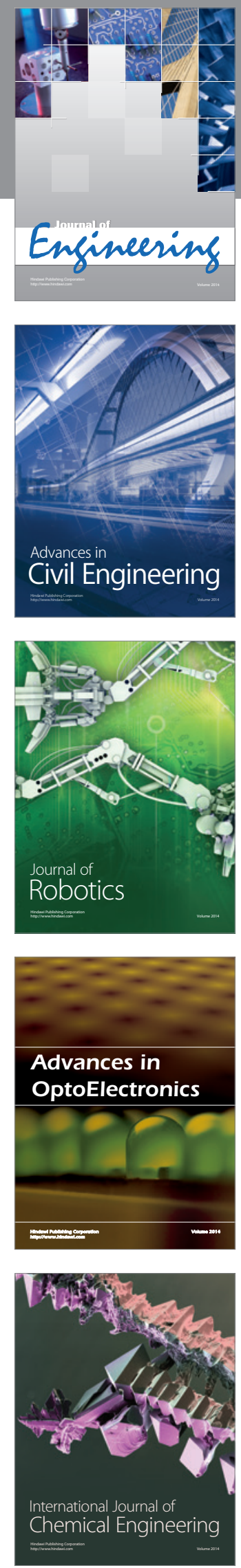

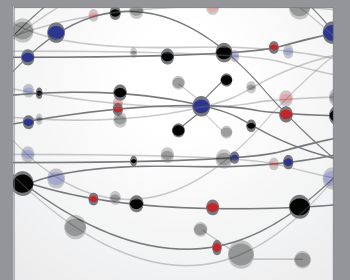

The Scientific World Journal
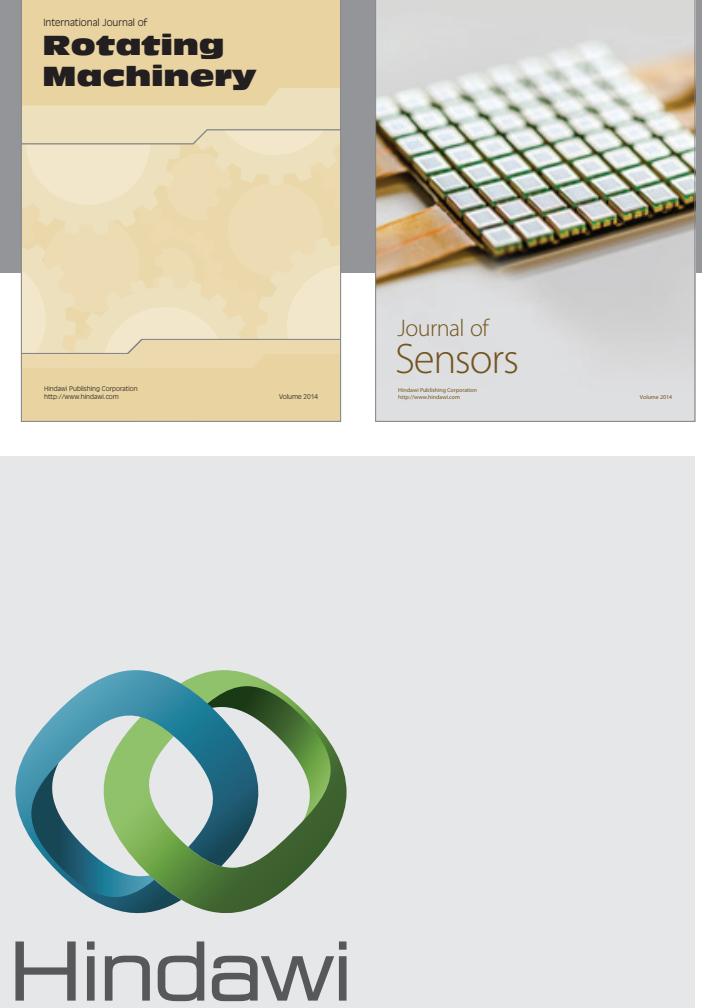

Submit your manuscripts at http://www.hindawi.com
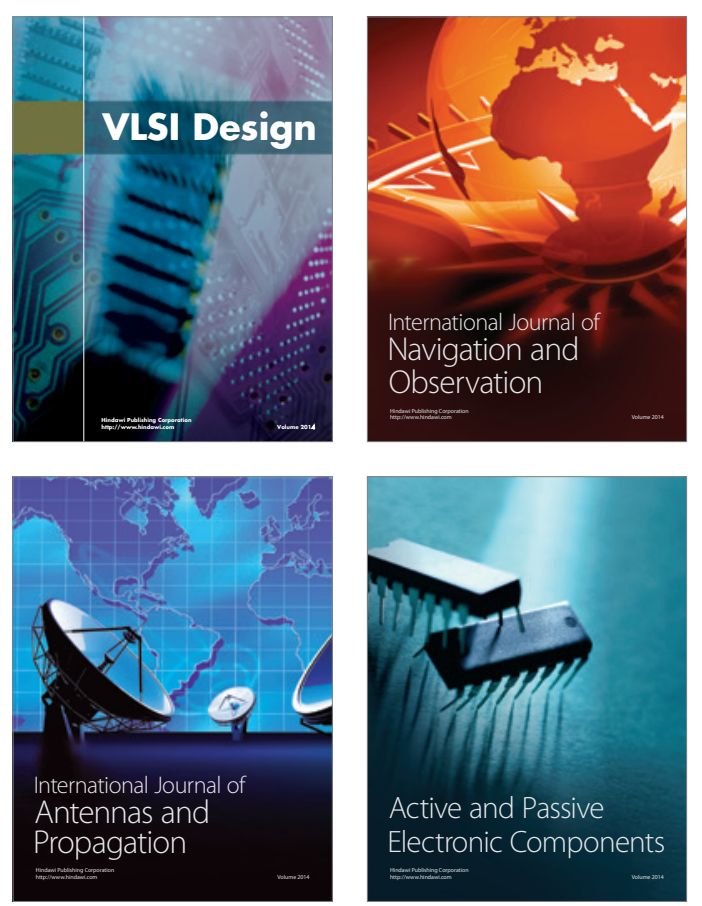
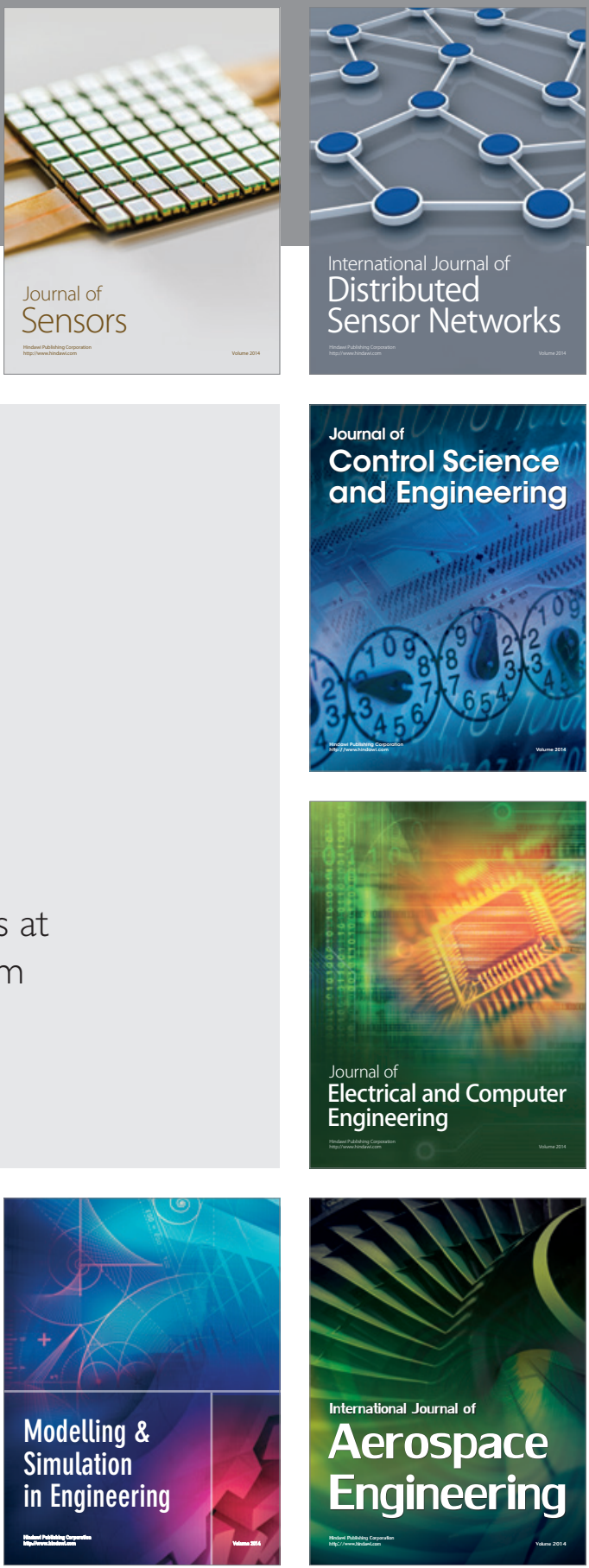

Journal of

Control Science

and Engineering
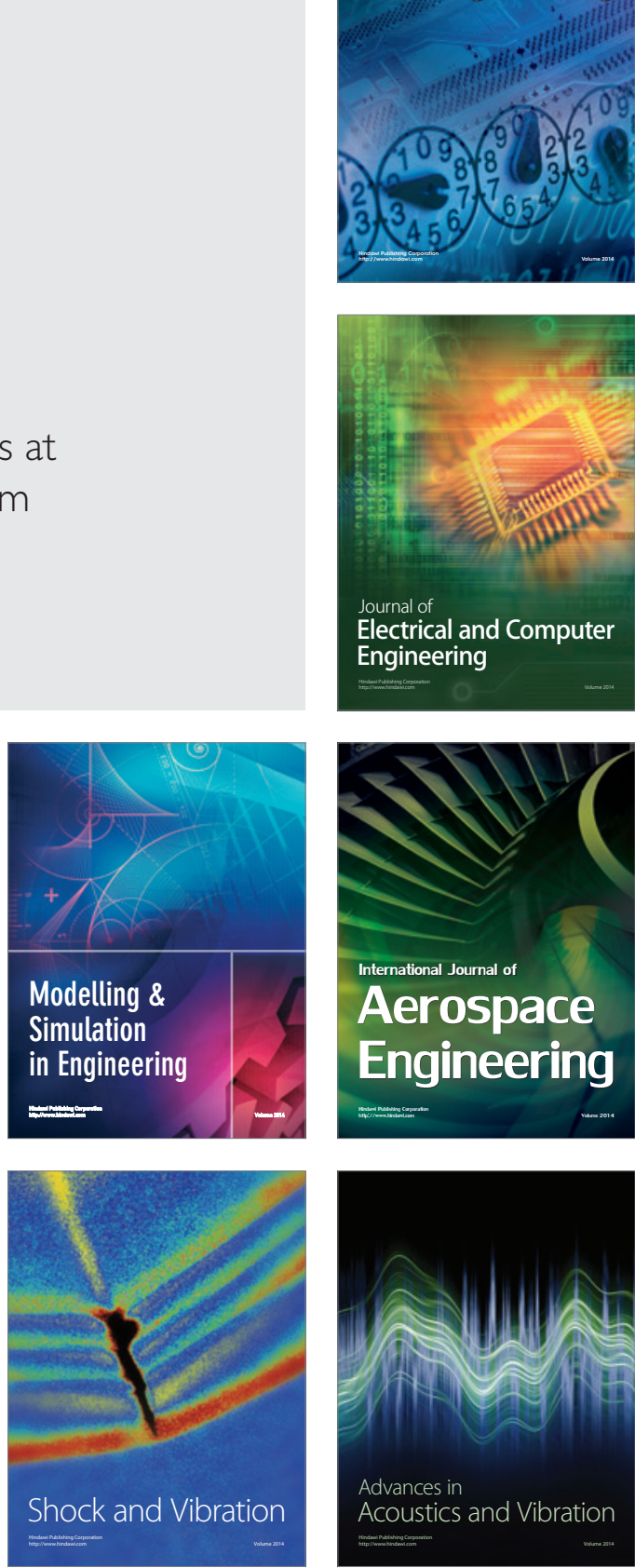